\title{
Personalized Medicine in the COVID-19 Vaccination Amid the Wake of Diseases Comorbidity Using Nigeria as a Case Example
}

\author{
Pius $\mathrm{M}^{1 *}$, Adebisi YA ${ }^{2}$, Muhammed $\mathrm{Y}^{3}$, Oladipo $0^{4}$, Ejim $\mathrm{D}^{5}$ and Dauda $\mathrm{AM}^{6}$ \\ ${ }^{1}$ Department of Medical Genetics, University of Cambridge, UK \\ ${ }^{2}$ Global Health Focus, Nigeria \\ ${ }^{3}$ Department of Biochemistry, Federal University Gusau, Nigeria \\ ${ }^{4}$ Department of Human Nutrition and Dietetics, University of Ibadan, Nigeria \\ ${ }^{5}$ Department of Biomedical Science, University of Chester, England \\ ${ }^{6}$ Faculty of life science, University of Bradford, England
}

\section{Review Article \\ Volume 5 Issue 4}

Received Date: November 22, 2021

Published Date: December 09, 2021

DOI: $10.23880 /$ eij-16000211

*Corresponding author: Mkpouto Pius, Department of Medical Genetics, University of Cambridge, UK, Tel: 08064110844; Email: piusmkpouto@gmail.com

\section{Abstract}

Since the onset of the pandemic, there have been over 200 million cases of COVID-19 cases reported and nearly 5 million deaths worldwide. The USA has been hardest hit with over 35 million cases and 713,350 deaths, followed by Brazil and India which suffered huge mortalities during the third wave. Regardless of the number of cases and consequent deaths recorded, no country has been immune to the economic downturn that the pandemic unleased, accentuating the need for widespread vaccination to reduce the impact of the virus. As at the time of writing this paper, the global vaccination stat report that 3.75 billion people have received at least a dose and 2.8 billion people are fully vaccinated worldwide. Nigeria is a developing country and like many third world countries was dependent on the developed countries for al protective equipment, test kits, as well as vaccines. The population in Nigeria has more youth than the elderly and explanation for why the cases reported were far less even with a population of over 200 million people. A little over 200,000 cases have so far been reported in Nigeria with about 2,837 deaths. An abysmally low $2.5 \%$ of the population have currently been vaccinated with only $1.1 \%$ having received both doses, owing to several reasons, and the death toll continues to count. Globally, the vaccination strategy has been to get the jabs to the most vulnerable first and then make it available to the general population. In this article, we discuss the relevance of personalized medicine in COVID-19 vaccination in a country, Nigeria, burdened with diseases comorbidity.

Keywords: Population; Vaccination; Coronavirus; Pandemic; Hypertension

Abbreviations: COVID-19: Coronavirus Disease 2019; SARS-CoV-2: Severe Acute Respiratory Syndrome Coronavirus 2; ACE-2: Angiotensin-Converting Enzyme 2; TMPRSS-2: Transmembrane Protein Serine 2; RBD: Receptor Binding Domain; COPD: Chronic Obstructive Pulmonary Disorder; ARBs: Angiotensin Receptor Blockers;
AR: Androgen Receptor; NSCR: National System of Cancer Registries; NCDs: National Survey of Non-Communicable Diseases; T2DM: Type 2 Diabetes Miletus; REMAH: Removing the Mask on Hypertension; NPHCDA: National Primary Healthcare Development Agency. 


\section{Epidemiology International Journal}

\section{Mechanism of COVID-19 Pathogenesis and Diseases Comorbidity}

The coronavirus disease 2019 (COVID-19) which accelerated to pandemic stage by March 2020 is caused by the severe acute respiratory syndrome coronavirus 2 (SARS-CoV-2). Characteristic of coronaviruses, SARS-CoV-2 possesses a spike glycoprotein on its cell surface which acts as its primary antigen and accelerates entry into target cells by binding to receptors on host cell surfaces [1]. The virus is an enveloped beta coronavirus which has a single-stranded 30 kilobase positive-sense RNA that encodes the 4 structural proteins namely, the spike (S) protein, membrane "matrix" (M) protein, Nucleocapsid (N) protein, and the envelope (E) protein [2]. The SARS-CoV-2 infection can be transmitted via respiratory droplet such as those emitted when breathing, sneezing, normal speech, and coughing. Therefore, the maintenance of physical distancing is important in reducing the spread of the disease. The infection could also be transmitted through contaminated surface and objects, which is why the use of hand sanitizer is essential is fighting the pandemic [3]. Other studies have reported that the virus could be present in body fluids such as saliva [4].

In typical coronavirus fashion, SARS-CoV-2 binds to the angiotensin-converting enzyme 2 (ACE-2) receptor located on epithelial cells. This binding is mediated by the spike protein which has a strong affinity for ACE-2 receptor and the binding of both molecules signals the transmembrane protein serine 2 (TMPRSS-2) which mediates proteolytic cleavage and facilitates viral entry by priming the virus for interaction with ACE-2 [5,6]. Upon entry into the host cell, the virus uncoats itself and then utilizes the host genetic and protein machinery to produce full-length antisense genome using its single stranded RNA as a template [7]. The spike protein is a trimer with 2 subunits namely SI and S2. The SI subunit contains 3 receptor-binding units including a receptor binding domain (RBD) which locks on to the ACE2 receptor, while the $S 2$ contains heptapeptide sequence 2 and $1[8,9]$. ACE-2 is a membrane-bound protein expressed in multiple human organs including the kidney, lungs, and in systems such as the central nervous system [10]. It is an important signal protein for the entry of SAR-CoV-2 into the host cell. Accelerated production of the receptor is reported in several pathological conditions. In diabetics there is an increased level of ACE- 2 receptors followed by a proportionate increase in Furin levels [11,12]. Furin is a type-1 membrane-bound proprotein convertase which activates the S protein by cleaving it into discrete S1 and S2 subunits thereby facilitating attachment to ACE- 2 receptors and evasion of the host immune system [13].

Elevated levels of these proteins are also observed in patients with chronic obstructive pulmonary disorder
(COPD) [14]. ACE-2 inhibitors, angiotensin receptor blockers (ARBs), and aldosterone receptor antagonists used in treating hypertension, cardiovascular and kidney disease result in upregulation of ACE- 2 receptors [15]. The binding and entry of SARS-CoV-2 into a host is mediated by interaction of Spike protein with ACE2 receptor, this interaction depends on host protease (such as cathepsin B and L, TMPRSS2, and furin processing [5]. The mechanism used by those proteases in mediating host entry is unclear [16]. But some studies have suggested that the TMPRSS2 mediate cell entry by the activation of membrane fusion protein through irreversible conformational change. Therefore, the TMPRSS2 is one of the studied androgen-regulated genes associated with prostate cancer [17].

The mechanism of TMPRSS2 in the mediation of prostate cancer is by promoting oncogenic transcription. More than half of all prostate cancers in men of European ancestry harbor a gene fusion that juxtaposes the androgen receptor (AR) regulatory promoter/enhancer elements of TMPRSS2 in front of the ETS family transcription factors, most commonly ERG [18]. Even though the regulation of ERG is not mediated by androgen, but this somatic gene rearrangement results in oncogenic ERG expression under androgen receptor signalling. Through the activation of HGF, TMPRSS2 promotes metastatic prostate cancer [19]. The metalloprotease ACE2 receptor (zinc dependent) is expressed in heart, kidney, and testis at high level [10]. Hence, the mechanism of governing TMPRSS2 and ACE2 expression could shed light on the disparity of SARS-CoV-2 mortality and morbidity in men [20]. While this introduction highlights the relationship between the SAR-COV-2 and disease comorbidities, the specific aim of this article, however, is to review personalised medicine for COVID-19 vaccination in Nigeria.

\section{Epidemiology of Common Chronic Diseases in Nigeria}

The resolution of epidemiological data in Nigeria often suffers due to a paucity of national disease registries, high buy-in to traditional medicine in rural areas, difficulty accessing the limited available health centres, the state of medical records (analogue, non-searchable) and other systemic issues. These challenges notwithstanding, this paper integrates data from high quality sources including National System of Cancer Registries (NSCR), National survey of non-communicable diseases (NCDs), Federal Ministry of Health and global agencies like the World Health Organisation's databases.

\section{Diabetes}

About 415 million people were estimated to be living with type 2 diabetes Miletus (T2DM) in the world in 2015 


\section{Epidemiology International Journal}

[21], a 400\% increase in incidence since 1980 according to data published by the WHO [22]. In this time, Africa has seen a 1.6 times faster rise in diabetes prevalence than the global average and now has higher prevalence rates of diabetes in its urban centres than some developed countries [23,24]. Nigeria is reported to have Africa's highest T2DM burden $[25,26]$ with a national survey of non-communicable diseases (NCDs) conducted in 1997 and a 2017 review of studies showing a prevalence rate of $2.2 \%$ and $4.1 \%$ respectively $[27,28]$. This represents a nominal doubling of prevalence, but since Nigeria's population grew from 113.5 million in 1997 to 191 million in 2017, the true burden of T2DM has grown $329 \%$ - from 2.38 million cases to 7.83 million - in the last two decades.

\section{Cancer}

Nigeria's cancer mortality in 2020 was figured at 78,899 with 124,815 new cases recorded [29]. This represents a modest increase from the 72,000 deaths and 102,000 new cases of cancers recorded in 2012 [30]. Breast cancer in the last decade has been the most prevalent, representing $22.7 \%$ of all new cases and $18.1 \%$ of all deaths 9 . The top 5 cancers of greatest burden in Nigeria are Breast (22.7\%), Prostate (12.3\%), Cervical (9.7\%), non-Hodgkin lymphoma (5.9\%) and colorectal cancer (5.2\%). The rates of Liver cancerwhich used to be the third most prevalent cancer but is now sixth - have fallen from $8 \%$ in 2012 to $4.2 \%$ in 2020.29 [31], The male to female death ratio was 1:2.2 and the peak age of death was between 51 and 60 years. It is unclear if this is due to a rise in detection of other kinds of cancer or a fall in the rate of Liver cancer (due to its higher mortality) as absolute number of cases have been relatively stable in that time.

\section{Hypertension}

The REMAH (Removing the Mask on Hypertension) study-Nigeria's largest and most recent nationwide study of the prevalence of pulmonary hypertension-established an alarming prevalence of the disease [32]. With an overall agestandardized prevalence of hypertension of $38.1 \%$, women had significantly higher rates at $41.8 \%$ vs men $31.8 \%$. A distinct regional divide was apparent from the data with the Northern regions registering about half the rate of the South; North-Central, 20.9\%; North-East, 27.5\%; North-West, 26.8\%; South-East, 52.8\%; South-South, 44.6\%; and SouthWest, $42.1 \%$. A rural-urban divide was absent/insignificant with $37.5 \%$ registered for rural areas versus $39.2 \%$ for urban areas. Yet, treatment rate significantly higher $(40.9 \%$ vs. $30.8 \%$ ), awareness better ( $62.2 \%$ vs. $56.6 \%)$ though control was similar (14\% vs. $10.8 \%$ ), in urban versus rural residents. Prior to the REMAH study, the 1997 NCD study was the last comprehensive nationwide investigation of hypertension, and it found the prevalence was $11.2 \% .27$ With the change in cut-off point for diagnosing hypertension to $140 / 90 \mathrm{mmHg}$ in 1999 by the WHO and International Society for Hypertension, the prevalence of hypertension was refactored by the Federal Ministry of Health in 2013 to stand at 20\% [33].

\section{Global and Nigeria's Vaccination Efforts}

Globally, the development of effective vaccines against the coronavirus has been fast with an impressive growth rate of the vaccine having the effect of putting the pandemic on a tight rein [34]. Development of vaccine in several research centres and pharmaceutical companies was announced as soon as SARS-CoV-2 was known to be its causative agent [35]. The effectiveness of a vaccine may be abused on people's strong preferences while a vaccine of low effect could also influence willingness of its acceptance by the population. To halt the COVID-19 pandemic, high vaccination coverage may be required hence the need to put into cognizance its demand in across countries where low- and middle-income countries (LMICs) or high-income countries [36]. By a year after the report of the first COVID-19 case, a minimum of 177 countries and territories had administered more than 878 million doses of a COVID-19 vaccine. Various strategies have been implored by countries with some vaccinating more of their citizens as fast as possible while others give precedence to populations more likely to be at risk making a sum of 879 million vaccines administered with 195 million fully vaccinated around the globe as at 15th April, 2021 [37].

Like any other African country, Nigeria had about 50 days or more to prepare for the pandemic since its outbreak in Wuhan, China. On March 12 2020, the Presidential Task Force on the fight against COVID-19 pandemic was inaugurated with the goal of National Response Strategy to help train healthcare workers on infection, prevention and control and respond to the pandemic [38]. Introduction of fiscal and monetary measures by the government coupled with partnerships from international agencies such as the World Bank, to mention a few are collaborating with the Taskforce to fight the coronavirus [39]. Despite disruptions associated with COVID-19 containment measures such as closure of borders, workplaces and educational institutions, restriction of movements (intra-urban/inter-urban transportation), negative responses have been recorded against the measures hence the reason for the continuous cases in the country. Nonchalant attitudes and failure to observe measures such as physical distancing and regular hand hygiene have caused a delay in flattening the curve of the pandemic hence birthing daily cases of infected individuals [40].

In March 2021, Nigeria received nearly 4 million doses of the AstraZeneca/Oxford vaccine shipped by COVAX facility from the Serum Institute of India (SII), Mumbai to Abuja, Nigeria with the aim to inoculate $70 \%$ of 200 million 


\section{Epidemiology International Journal}

people by 2022 [41]. As at 15th of April, 2021, a total of 1.08 million doses have been administered to the citizens [37]. To support the vaccine procurement, MTN, a telecommunication company also delivered 300,000 doses of the vaccine. According to a poll by the National Primary Healthcare Development Agency (NPHCDA), various reactions have being recorded in term of 'Vaccine hesitancy' with 50\% of the population showing interest in vaccine inoculation [42].

Before the arrival of the vaccine, vaccine accountability officers were appointed both in states and local government areas to ensure the vaccines are kept safe, potent and closely monitored through its management and utilization. The minimum criteria required for a vaccination program to hold include training, availability of data-gathering tools, cold storage, transport and logistics for healthcare workers [42]. Registration of people electronically for vaccination to ensure its efficiency and orderly schedule for its collection is being done in Nigeria through an e-registration portal under an electronic immunization data management scheme. According to pre-planned classifications which are in phases, frontline workers are prioritized for the vaccination followed by populations at high risk for severe diseases, essential workers and lastly the general public [43]. Being vaccinated does not mean that people can throw caution to the wind rather Nigerians need to continually adhere to other public health and social measures by wearing nose masks, washing of hands or use of hand sanitizers and maintenance of physical distancing to help curb the virus [41].

\section{Advancing Precision Medicine in the Era of COVID-19 Vaccination}

With the passage of time medical practice has enjoyed increased efficiency and one of such processes currently being maximised in the interest of improved patient management is personalised medicine. The term implies the incorporation of the unique genomic composition of each individual as well as the variability of their environment and lifestyle choices into the diagnosis of their disease condition and management of the condition [44]. A most important element of precision medicine is that it takes cognizance of the patient's preexisting conditions while making recommendations for preferential treatment pathways that may be more effective. This is most applicable to the distribution of COVID-19 vaccines given the time sensitive nature of the pandemic. The application of precision medicine to the vaccine distribution process will help identify patient groups with pre-existing conditions that make them more vulnerable to more grave reactions to COVID-19. Several disease conditions result in the weakening of the patient's immune system thus leaving them vulnerable to the SARS-COV-2.

Precision medicine affords the benefit of insight into what risk factors should have a greater weighting when considering who should be prioritised for COVID-19 vaccines. The most obvious groups of priorities are currently healthcare workers and people with debilitating disease conditions, but this prioritisation needs to be informed by factors other than physical assessment in order to ensure that the most vulnerable groups are adequately protected. Nigeria has high incidence of diabetes and hypertension which top the charts as comorbidities for COVID-19 [45]. The incidences of cancers in the country are also rapidly on the rise consistent with the global trend [46]. This makes it extremely expedient that a more specialised and bespoke approach be adopted to reflect these peculiarities.

\section{Conclusion}

Nigeria, with its characteristic high burden of disease comorbidities, will benefit immensely from personalized medicine in treatment or diseases prevention interventions such as vaccination. The emergence of COVID-19 and the need to vaccinate the adult population with various diseases comorbidities reemphasize the need to apply precision medicine to vaccination and disease treatment interventions. This is a wakeup call for Nigeria and other countries with high burden of disease to invest in precision medicine as there is no better time than now.

\section{References}

1. Huang Y, Yang C, Xu XF, Xu W, Liu SW (2020) Structural and functional properties of SARS-CoV-2 spike protein: potential antivirus drug development for COVID-19. Acta Pharmacologica Sinica 41(9): 1141-1149.

2. Naqvi AAT, Fatima K, Mohammad T, Fatima U, Singh IK, et al. (2020) Insights into SARS-CoV-2 genome, structure, evolution, pathogenesis and therapies: Structural genomics approach. Biochim Biophys Acta Mol Basis Dis 1866(10): 165878.

3. Falahi S, Kenarkoohi A (2020) Transmission routes for SARS-CoV-2 infection: review of evidence. New Microbes New Infect 38: 100778.

4. Anfinrud P, Bax CE, Stadnytskyi V, Bax A (2020) Could SARS-CoV-2 be transmitted via speech droplets?. MedRxiv.

5. Hoffmann M, Winkler HH, Smith JC, Krüger N, Sørensen LK, et al. (2020) Camostat mesylate inhibits SARSCoV-2 activation by TMPRSS2-related proteases and its metabolite GBPA exerts antiviral activity. BioRxiv pp: $1-42$.

6. Yan R, Zhang Y, Li Y, Xia L, Guo Y, et al. (2020) Structural 
basis for the recognition of SARS-CoV-2 by full-length human ACE2. Science 367(6485): 1444-1448.

7. Wrapp D, Vlieger DD, Corbett KS, Torres GM, Wang N, et al. (2020) Structural Basis for Potent Neutralization of Betacoronaviruses by Single-Domain Camelid Antibodies. Cell 181(5): 1004-1015.

8. Shang J, Wan Y, Luo C, Ye G, Geng Q, et al. (2020) Cell entry mechanisms of SARS-CoV-2. PNAS 117(21): 11727-11734.

9. Huang Y, Yang C, Xu X, Xu W, Liu S (2020) Structural and functional properties of SARS-CoV-2 spike protein: potential antivirus drug development for COVID-19. Acta Pharmacol Sinica 41: 1141-1149.

10. Gheblawi M, Wang K, Viveiros A, Nguyen Q, Zhong JC, et al. (2020) Angiotensin-Converting Enzyme 2: SARSCoV-2 Receptor and Regulator of the Renin-Angiotensin System. Circulation Research 126(10): 1456-1474.

11. Rao S, Lau A, So HC (2020) Exploring Diseases/Traits and Blood Proteins Causally Related to Expression of ACE2, the Putative Receptor of SARS-CoV-2: A Mendelian Randomization Analysis Highlights Tentative Relevance of Diabetes-Related Traits. Diabetes Care 43(7): 14161426.

12. Fernandez C, Rysä J, Almgren P, Nilsson J, Engström G, et al. (2018) Plasma levels of the proprotein convertase furin and incidence of diabetes and mortality. Journal of Internal Medicine 284(4): 377-387.

13. Ejaz H, Alsrhani A, Zafar A, Javed H, Junaid K, et al. (2020) COVID-19 and comorbidities: Deleterious impact on infected patients. Journal of Infection and Public Health 13(12): 1833-1839.

14. Wan Y, Shang J, Graham R, Baric RS, Li F (2020) Receptor Recognition by the Novel Coronavirus from Wuhan: an Analysis Based on Decade-Long Structural Studies of SARS Coronavirus. J Virol 94(7): e00127-e00130.

15. Bosso M, Thanaraj TA, Farha MA, Alanbaei M, Abubaker J, et al. (2020) The Two Faces of ACE2: The Role of ACE2 Receptor and Its Polymorphisms in Hypertension and COVID-19. Mol Ther Methods Clin Dev 18: 321-327.

16. Coutard B, Valle C, De Lamballerie X, Canard B, Seidah NG, et al. (2020) The spike glycoprotein of the new coronavirus 2019-nCoV contains a furin-like cleavage site absent in CoV of the same clade. Antiviral Res 176: 104742.

17. Afar DE, Vivanco I, Hubert RS, Kuo J, Chen E, et al. (2001) Catalytic cleavage of the androgen-regulated TMPRSS2

\section{Epidemiology International Journal}

protease results in its secretion by prostate and prostate cancer epithelia. Cancer Res 61(4): 1686-1692.

18. Tomlins S, Rhodes D, Perner S, Dhanasekaran S, Mehra $R$, et al. (2005) Recurrent fusion of TMPRSS2 and ETS transcription factor genes in prostate cancer. Science 310(5748): 644-648.

19. Lucas JM, True L, Hawley S, Matsumura M, Morrissey C, et al. (2008) The androgen-regulated type II serine protease TMPRSS2 is differentially expressed and mislocalized in prostate adenocarcinoma. J Pathol 215(2): 118-125.

20. Deng Q, Rasool RU, Russell RM, Natesan R, Asangani IA (2021) Targeting androgen regulation of TMPRSS2 and ACE2 as a therapeutic strategy to combat COVID-19. iScience 24(3): 102254.

21. (2015) IDF diabetes atlas. International Diabetes Federation, Brussels, Belgium.

22. Roglic G (2016) WHO Global report on diabetes: A summary. IJNCD 1: 3-8.

23. Beran D, Yudkin JS (2006) Diabetes care in sub-Saharan Africa. Lancet 368(9548): 1689-1695.

24. Guariguata L, Whiting DR, Hambleton I, Beagley J, Linnenkamp U, et al. (2014) Global estimates of diabetes prevalence for 2013 and projections for 2035. Diabetes Res Clin Pract 103(2): 137-149.

25. Mbanya JCN, Motala AA, Sobngwi E, Assah FK, Enoru ST (2010) Diabetes in sub-Saharan Africa. Lancet 375(9733): 2254-2266.

26. Ogbera AO, Ekpebegh C (2014) Diabetes mellitus in Nigeria: The past, present and future. World J Diabetes 5(6): 905-911.

27. Akinkugbe 00 (1997) Non-Communicable Diseases in Nigeria National Survey (Final Report) on Hypertension, Coronary Heart Disease, Diabetes Mellitus, Haemoglobinopathies, G6PD Deficiency and Anaemia. National Expert Committee on Non-Communicable Diseases. Federal Ministry of Health and Social Services, Lagos. References, Scientific Research Publishing.

28. Adeloye D, Ige JO, Aderemi AV, Adeleye N, Amoo EO, et al. (2017) Estimating the prevalence, hospitalisation and mortality from type 2 diabetes mellitus in Nigeria: a systematic review and meta-analysis. BMJ Open 7(5): e015424.

29. Ferlay J, Colombet M, Soerjomataram I, Parkin DM, Piñeros M, et al. (2021) Cancer statistics for the year 
2020: An overview. Int J Cancer 149(4): 778-789.

30. Atuwo D, Amedu J, Adewole I (2018) Nigeria National Cancer Control Plan 2018-2022. National Cancer Control Program, pp: 1-67.

31. Akintola A, Odutola M, Olayinka T, Akinjiola A, Nwokwu U, et al. (2016) Cancer in Nigeria 2009-2016. Nigerian National System of Cancer Registries, pp: 1-206.

32. Odili AN, Chori BS, Danladi B, Nwakile PC, Okoye IC, et al. (2021) Prevalence, Awareness, Treatment and Control of Hypertension in Nigeria: Data from a Nationwide Survey 2017. Glob Heart 15(1): 47.

33. (2013) National Policy and Strategic Plan of Action on Prevention and Control of Non-Communicable Diseases (NCDs). Federal Ministry of Health, WHO, pp: 1-2.

34. Marcus JS (2021) Has the European Union squandered its coronavirus vaccination opportunity? Bruegel.

35. Sun X, Wagner AL, Ji J, Huang Z, Zikmund Fisher BJ, et al. (2020) A conjoint analysis of stated vaccine preferences in Shanghai, China. Vaccine 38(6): 1520-1525.

36. Wagner AL, Boulton ML, Sun X, Mukherjee B, Huang Z, et al. (2017) Perceptions of measles, pneumonia, and meningitis vaccines among caregivers in Shanghai, China, and the health belief model: a cross-sectional study. BMC Pediatr 17(1): 143.

37. Pettersson H, Manley B, Hernandez S, McPhillips D, Arias T (2021) Tracking Covid-19 vaccinations worldwide. CNN Health.

\section{Epidemiology International Journal}

38. Ibeh IN, Enitan SS, Akele RY, Isitua CC, Omorodion F (2020) Global Impacts and Nigeria Responsiveness to the COVID-19 Pandemic. International Journal of Healthcare and Medical Sciences 6(4): 27-45.

39. (2021) Nigeria Scales Up Its COVID-19 Vaccination with New Funding for Vaccine Purchase and Deployment. World Bank.

40. Amimo F, Lambert B, Magit A, Hashizume M (2021) A review of prospective pathways and impacts of COVID-19 on the accessibility, safety, quality, and affordability of essential medicines and vaccines for universal health coverage in Africa. Globalization and Health 17: 42.

41. (2021) COVID-19 vaccines. WHO Regional Office for Africa.

42. Leon U (2021) Nigeria: COVID-19 vaccine rollout kicks off in Africa's most populous country. Africa Renewal.

43. (2021) COVID-19 Vaccine Phases and Planning. Minnesota Dept. of Health.

44. (2018) What Are Precision Medicine and Personalized Medicine?. Health IT Analytics.

45. Ovosi OB, Asuke S, Abdulrahman SO, Ibrahim MS, Ovosi JO, et al. (2018) Prevalence and correlates of hypertension and diabetes mellitus in an urban community in NorthWestern Nigeria. Pan Afr Med J 29: 97.

46. Uchendu OJ (2020) Cancer Incidence in Nigeria: A Tertiary Hospital Experience. Asian Pacific Journal of Cancer Care 5(1): 27-32. 